\title{
A Hop-by-Hop Multipath Routing Protocol Using Residual Bandwidth for Wireless Mesh Networks
}

\author{
Eun-Joo Oh, Sungil Lee, and Jae-Sung Lim \\ Graduate School of Information and Communications, Ajou University \\ San 5, Wonchun-dong, Youngtong-gu, Suwon, 447749, South Korea \\ \{oezoo, openlsi, jaslim\}@ajou.ac.kr
}

\begin{abstract}
In wireless mesh networks, there are mesh routers which can compose a wireless backbone with low mobility. We propose a hop-by-hop multipath routing scheme which is suitable for mesh routers offering network reliability with route redundancy. We extend DSDV in order to have multiple next hops to all nodes in the network without additional overheads. The basic idea of our scheme is to make several paths between the source and destination by selecting a proper next hop at every forwarding data. We choose a mesh router with the highest residual bandwidth as a next hop among multiple ones. Through periodic one hop broadcasting, not only we can get residual bandwidth information but also we can detect route failures fast and reduce the number of routing overhead packets. Through simulation, we represent that our scheme is more efficient than DSDV in delivering data to the destination when traffic is heavy, reducing overhead packets in the network, and preventing data loss when the route failure occurs.
\end{abstract}

Keyword: Wireless mesh networks, Routing, Proactive routing, Hop-by-Hop multipath, Residual bandwidth.

\section{Introduction}

As the use of Internet is increased, the demand to utilize Internet wherever and whenever is also increased. Because the size of devices is getting smaller and the capacity of them is getting better, they can satisfy the increasing demand for Internet. In these points of view, wireless mesh networks come. Wireless mesh networks take charge of connections both between the same networks and among the different networks. This fact makes it possible to use any data located in Internet or other networks whenever people want to. Wireless mesh networks consist of mesh routers and mesh clients. Mesh routers act as bridges which connect to different networks, gateways which connect to the Internet, and a wireless backbone. Mesh clients, as wireless terminals, can be hosts and routers like nodes in Ad-Hoc networks. Mesh clients also perform as a ad hoc gateway in order to connect to the wireless mesh backbone to access Internet[1].

We find out that mesh routers have similar characteristics to the nodes in Ad-Hoc networks such as wireless multi-hop communication. However, the mesh routers have 
different features in that they have very low mobility and no energy constraint, and they form a wireless infrastructure backbone including the bridging and gateways functions. The main data in wireless mesh networks must be the data from or to Internet through gateways. Moreover, it is expected that there are a lot of data traffics among mesh routers, especially audio and video traffics which are sensitive of a time and should satisfy QoS(Quality of Service) requirement. Therefore, a new routing protocol is necessary among mesh routers to reflect these unique characteristics and it should be different from the existing routing protocols in Ad-Hoc networks.

A lot of routing protocols has been studied in Ad-Hoc networks actively[2]-[9]. Routing is a very challenging task in Ad-Hoc networks because the Ad-Hoc networks have characteristics such as the unpredictability of environment due to node failure, the unreliability of wireless medium, resource-constrained nodes, and dynamic topology due to mobility. Ad-Hoc routing protocols are divided largely into two parts according to the time when a routing path is determined. The routing path is calculated whether before or at transmitting data, which are proactive protocols (DSDV[4], OLSR[5]) and reactive protocols(DSR[6], AODV[7]). The proactive protocols are also called table-driven schemes and they calculate the routing path before transmitting data. Each node in the network exchanges its routing table periodically and it can know network information such as the topology, the link state, and the routes. After setting up the routing table, the nodes can know the path to all nodes in the network and they can send data immediately whenever data to send is occurred. The reactive protocols are also called on-demand schemes and they calculate the routing path at transmitting data. Because these schemes calculate the path to the destination only when data to send is occurred, they don't have to exchange the routing tables periodically. They can reduce the number of overhead packets but the end-to-end delay is increased because data are able to be sent after calculating the routing path[2], [3].

In Ad-Hoc networks, DSDV[4] is a renowned proactive routing protocol. As a distance vector scheme, DSDV selects a next hop which has minimum hop counts to the destination. By exchanging routing tables, each node in the network can know the distance information as hop counts and next hops for all other nodes in the network with the minimum distance. As a proactive routing scheme, DSDV updates the routing table periodically. There are two ways to update the routing table, one, called a full dump, will carry all of the available routing information. The other, called an incremental, will carry only information changed since the last full dump. When the routing table is updated, routes are always preferred if the sequence numbers are newer and if the sequence numbers are the same and yet the lower hop count is better. The sequence number prevents the formation of loop because the route which has newer sequence number is preferred.

To compensate for the dynamic and unpredictable nature of Ad-Hoc networks, multipath routings are studied actively, too[9]. Multipath routing allows the establishment of multiple paths between a single source and single destination node. Load balancing can be achieved by spreading the traffic along multiple routes. If multiple paths are used simultaneously to route data, the aggregate bandwidth of the paths may satisfy the bandwidth requirement of the application. Since there is more bandwidth available, smaller end-to-end delay may be achieved. In [10], it is showed 
that a distance vector routing could be extended to offer the computation of all possible alternative paths with instance loop freedom.

Proactive routing protocols are proper for wireless mesh networks especially among mesh routers due to the mesh routers' characteristics. As a wireless backbone, the traffic patterns of mesh routers are likely that a large subset of nodes communicate with each other and the source and destination pairs are also changing with time. If mesh routers know all network information, they can minimize end-to-end delay by sending data without calculating a path to destination. In multi-hop wireless networks, as hop counts to traverse increase, the throughput is sharply decreased. This has been confirmed by several simulation studies based on 802.11 and other MAC(Medium Access Control) protocols similar to 802.11[12], [13]. We prefer DSDV because it is one of the well-known proactive routings and selects a next hop with minimum hop counts to the destination. However, it is hard to offer networks route redundancy for reliable data transmission because DSDV maintains just one next hop to each destination. Generating a lot of routing overhead packets is also a weak point of the proactive routing schemes to declare and update routing tables. If the data transmitting route gets into trouble, DSDV takes much time to recover the route and causes a lot of data loss. QoS metrics such as the bandwidth, the link state, and the queue state are hard to apply to DSDV because it is difficult to come up with network's information changed rapidly through periodic routing table updates.

In this paper, we propose the hop-by-hop multipath scheme of extending DSDV and adding a neighbor table containing residual bandwidth information. By extending DSDV, mesh routers can get multiple next hops without any additional packets or calculations. The multiple next hops should have minimum hop counts to all destinations. They enable our proposed scheme to operate as the hop-by-hop multipath based on minimum distance. Mesh routers make and maintain a neighbor mesh routers' table by using a HELLO message containing residual bandwidth information. We calculate the residual bandwidth according to [11] using HELLO bandwidth estimation. When a mesh router transmits data, it selects one of the multiple next hops according to the residual bandwidth information in the neighbor table, larger bandwidth value is preferred. Through the periodic broadcasting of HELLO messages, mesh routers can detect the state of topology fast. If the mesh router does not take periodic HELLO messages from neighbor routers, it concludes that the neighbor router has problem and it changes the next hop with another one to the destination directly without any additional procedure. The HELLO message also can make routing table updates operated only when there is no route to the destination. Our proposed scheme can deliver a lot of data to the destination by distributing data to the network using multiple next hops and residual bandwidth information especially when the data traffic is heavy. It can also reduce the number of routing overhead packets by modifying the routing update scheme of DSDV with the neighbor table, and prevent a lot of data loss by detecting problem quickly and changing a next hop directly.

This paper is organized as follows: In Section 2, we introduce our proposed routing scheme. In Section 3, we represent our simulations and discuss the performance results obtained from a series of simulations. In Section 4, we will make conclusions and future research. 


\section{Proposed Scheme: HMP Routing Protocol}

In this paper, we propose a new routing protocol suitable for mesh routers. We call the proposed scheme HMP(Hop-by-hop MultiPath) routing protocol. The HMP has two routing tables: a forwarding table and a neighbor table. The forwarding table is a routing table similar to DSDV's but it has multiple next hop information to all the other mesh routers in the network with minimum hop counts. The neighbor table is a table which stores neighbor routers' residual bandwidth information and it is used to choose a next hop and come up with the change of network state.

\subsection{Forwarding Table: Multiple Next Hops}

We modify DSDV because it has relatively little overhead among proactive routing protocols and it is simple to be extended to a multipath scheme. We represent a new approach to add a multipath capability to DSDV. We modify DSDV's routing table in order to take multiple next hop information to the destination with minimum hop counts. The basic idea of our scheme is to make several paths between a source and destination by selecting a proper next hop among multiple ones at every forwarding data, as a hop-by-hop multipath scheme.

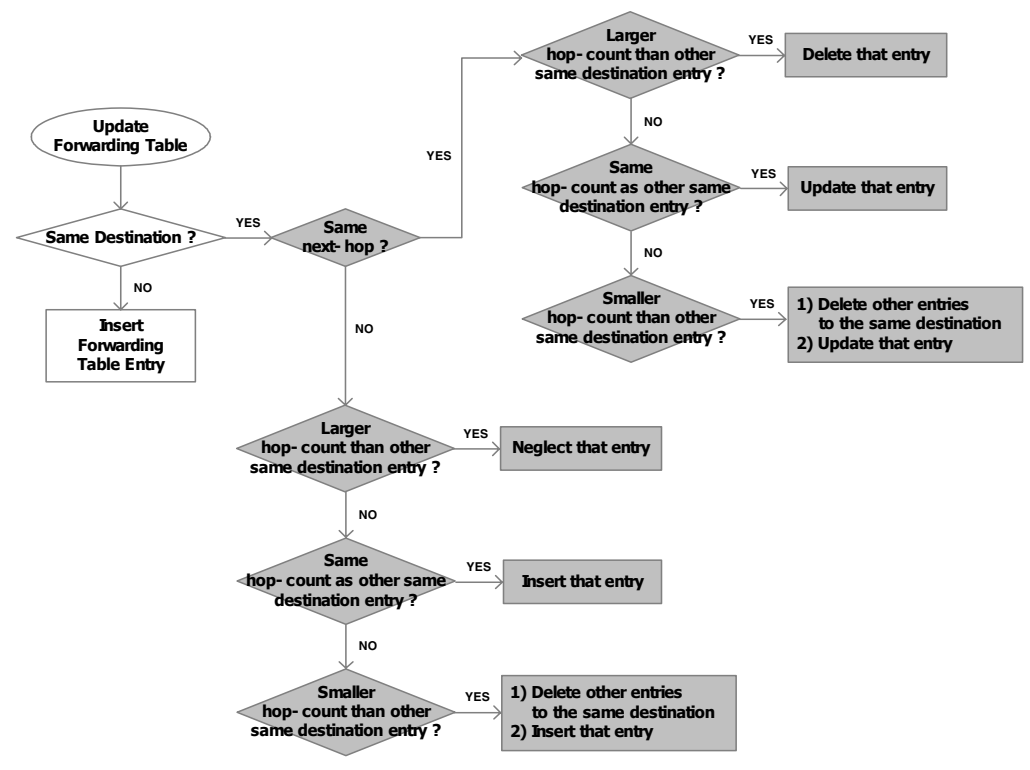

Fig. 1. Procedure of Forwarding Table Update

Figure 1 shows the way of modifying the procedure of DSDV to get multiple next hop information to all nodes in the network without any additional routing overheads. When exchanging routing tables, if the received updating message has the same sequence number but it is from a different mesh router, the message is used to update a forwarding table. Even though the incoming information has the same destination, it 
is determined whether to be used or not according to the next hop information. This way gives multiple next hops to the routing table. After checking the next hop information, it checks hop counts. According to the hop counts, the update procedure is changed as shown in figure 1 . This way makes the routing table get the minimum hop counts to the all destinations. Without any additional routing messages or calculations, we can get the forwarding table containing multiple next hops to all mesh routers with the minimum hop counts.

Our proposed multipath scheme can distribute data all over the network and achieve the load balancing using next hops properly. This distributing feature can prevent the occurrence of bottleneck mesh routers, especially when the amount of data is increased all over the network. Therefore, our scheme improves the efficiency of data delivery. Our proposed scheme can also improve the reliability of data transmission with route redundancy. When a mesh router in the route gets some problem such as link or route failures, one of other next hops can be used immediately without any other procedure to recover the route. Therefore, our proposed scheme decreases a lot of data loss when the route has problem.

Once forming topology among the mesh routers, the topology is hardly changed due to low mobility and the routing table's information can be used for relatively long. Therefore, the periodic routing table update in DSDV is not quite necessary. We modify the routing table update so that the update is performed only when a new mesh router joins or leaves the network. Even though the topology is changed, we can delay the routing table update until there is no route to the destination in order to reduce overhead packets more. Because our proposed scheme has multiple routes to the destination, the routing tables are updated only when there is no route to the destination. This modification can reduce a great number of routing overhead packets.

\subsection{Neighbor Table: Residual Bandwidth and Topology Control}

We propose making a neighbor table through HELLO messages to store residual bandwidth information of neighbor mesh routers and control topology. The HELLO message contains residual bandwidth information and it is exchanged between onehop neighbor routers through a periodic broadcasting. Being different from the forwarding table, the neighbor table maintains the information of just one hop neighbor. The information of the neighbor table is used to select a next hop out of multiple next hops when transmitting data. The mesh router with the maximum residual bandwidth is selected as a next hop. This means that we select a path with higher bandwidth among several paths keeping minimum hop counts.

We calculate the residual bandwidth according to [11] as the raw link capacity minus the overall consumed bandwidth, divided by a weight factor. It is necessary to divide the residual bandwidth by the weight factor due to IEEE 802.11 MAC's nature which RTS(Request To Send), CTS(Clear To Send), and ACK(ACKnowledgement) packets consume bandwidth, the back-off scheme cannot fully use the entire bandwidth, and packets can collide, resulting in packet retransmissions. Each mesh router broadcasts its residual bandwidth information periodically calculating through above method. We think that if a mesh router has more residual bandwidth, the router has more ability to manage data efficiently and the data can be transmitted with higher transmission rate. Therefore, selecting a next hop which has a maximum 
residual bandwidth basically minimum hop counts helps the network use the capacity of 802.11 WLAN(Wireless Local Area Network) evenly, reduce waste of network bandwidth due to reducing retransmission, distribute data to the router with more data processing capacity, and prevent a network from occurring bottleneck mesh routers.

We can use the neighbor table to control topology by finding out the routers' state. The neighbor table also makes it possible to update the forwarding table only when the network topology is changed. If neighbor router's information is not updated for a certain time, we can conclude that the mesh router already leaved the network or the router has a problem. Then, the router sets the residual bandwidth of the neighbor router zero in order not to select the router as a next hop. It can send data through another next hop directly. If the residual bandwidth of all possible next hops is zero, which means that there is no route to get to the destination, the forwarding table update is started through all over the network. At this moment, the entries that have zero residual bandwidth in the neighbor table are deleted. This method reduces the amount of data loss by using another next hop directly and it can also reduce the number of routing overhead packets not by updating routing tables immediately and periodically all over the network.

The interval of broadcasting HELLO messages is thoroughly related to the time to know a route failure and a topology change. If the interval is short, the time to detect a route failure is also short but the number of overhead packets is increased. On the other hand, if the interval is long, the time to detect a route failure is also long but the number of overhead packets is decreased.

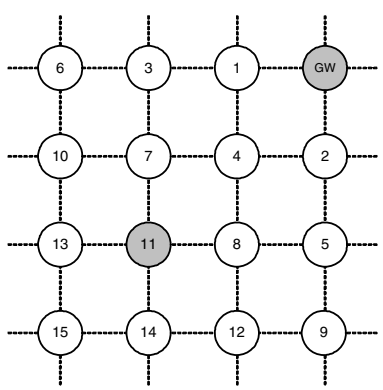

(a) Example Topology of $4 \times 4$ Grid

\begin{tabular}{|c|c|c|}
\hline \multicolumn{3}{|c|}{ Neighbor Table of $\mathbf{1 1}$} \\
\hline Nghr. & R_BW_ & $\ldots$ \\
\hline 7 & $5 \mathrm{M}$ & $\ldots$ \\
\hline 8 & $10 \mathrm{M}$ & $\ldots$ \\
\hline 13 & $\mathbf{8} \mathrm{M}$ & $\ldots$ \\
\hline 14 & $\mathbf{8} \mathrm{M}$ & $\ldots$ \\
\hline
\end{tabular}

(c) Neighbor Table

\begin{tabular}{|c|c|c|c|}
\hline \multicolumn{4}{|c|}{ Forwarding Table of 11} \\
\hline Dst. & Nxt_Hp & Hp_Cnt & $\ldots$ \\
\hline \multirow{2}{*}{ GW } & 7 & 4 & $\ldots$ \\
\hline & 8 & 4 & $\ldots$ \\
\hline \multirow{2}{*}{1} & 7 & 3 & $\ldots$ \\
\hline & 8 & 3 & $\ldots$ \\
\hline \multirow{2}{*}{2} & 7 & 3 & $\ldots$ \\
\hline & 8 & 3 & $\ldots$ \\
\hline 3 & 7 & 2 & $\ldots$ \\
\hline \multirow{2}{*}{4} & 7 & 2 & $\ldots$ \\
\hline & 8 & 2 & $\ldots$ \\
\hline 5 & 8 & 2 & $\ldots$ \\
\hline \multirow{2}{*}{6} & 7 & 3 & $\ldots$ \\
\hline & 13 & 3 & $\ldots$ \\
\hline 7 & 7 & 1 & $\ldots$ \\
\hline 8 & 8 & 1 & $\ldots$ \\
\hline \multirow{2}{*}{9} & 8 & 3 & $\ldots$ \\
\hline & 14 & 3 & $\ldots$ \\
\hline \multirow{2}{*}{10} & 7 & 2 & $\ldots$ \\
\hline & 13 & 2 & $\ldots$ \\
\hline 11 & 0 & 0 & $\ldots$ \\
\hline \multirow{2}{*}{12} & 8 & 2 & $\ldots$ \\
\hline & 14 & 2 & $\ldots$ \\
\hline 13 & 13 & 1 & $\ldots$ \\
\hline 14 & 14 & 1 & $\ldots$ \\
\hline \multirow{2}{*}{15} & 13 & 2 & $\ldots$ \\
\hline & 14 & 2 & $\ldots$ \\
\hline
\end{tabular}

(b) Forwarding Table

Fig. 2. Example Tables of Proposed Scheme 
Figure 2 shows an example of a forwarding table and a neighbor table as a result of operating the proposed scheme in $4 \times 4$ grid topology. Let's assume that the router 11 wants to send data to the GW. First, the router 11 sees the forwarding table to find a next-hop. Next, the router 11 can know there are two next hop candidates, router 7 and 8 . Then, the router 11 sees the neighbor table to determine which next hop has more residual bandwidth. Finally, the router 11 chooses the router 8 as a next hop and sends data through the router 8 . In case that the router 8 leaves the network, the router 11 can not take the HELLO message from router 8 . The router 11 sets the residual bandwidths of router 8 zero in the neighbor table. Then, the router 11 sends data to the GW through the router 7 . Because the residual bandwidths of the router 8 become zero the router 11 does not choose the router 8 as a next hop.

\section{Simulations}

We evaluated the proposed scheme through NS-2. The simulation was conducted with the following parameters. The simulation topology is the same as figure 2(a).

Table 1. Simulation Parameters

\begin{tabular}{|c|c|}
\hline Parameter & Value \\
\hline Topology & $4 \times 4$ grid topology \\
\hline The number of nodes & 16 \\
\hline Distance between nodes & $50 \mathrm{~m}$ \\
\hline Transmission range & $50 \mathrm{~m}$ \\
\hline Traffic & FTP \\
\hline Packet size & $64 \sim 2048$ bytes \\
\hline MAC protocol & $802.11 \mathrm{WLAN}$ \\
\hline Link bandwidth & $11 \mathrm{Mbps}$ \\
\hline The broadcasting interval of HELLO message & 10 seconds \\
\hline Simulation time & $200 \sim 300$ seconds \\
\hline
\end{tabular}

Figure 3 shows the total throughput with increase of the packet size. Figure 4 shows the average delay according to the number hops to traverse when the size of packet is 1024 bytes. In the simulation of figure 3 and 4, routers $2,3,8,10,14,15$, and GW participate in data communication showed in figure 2(a). The packet size is increased with the following 64, 128, 256, 512, 1024, and 2048 bytes.

We can see that HMP shows better performance of throughput and delay than DSDV in all cases in figure 3 and 4. As the size of packet increases, the throughput gap between HMP and DSDV gets wide. Similarly, as the hop counts to traverse increases, the delay gap between HMP and DSDV gets wide, too. The reason why HMP has better performance is that HMP can distribute data to the networks evenly using multiple next hops properly based on the residual bandwidth information. HMP achieve better performance of the total throughput and the average delay when there are a lot of data in the network. 


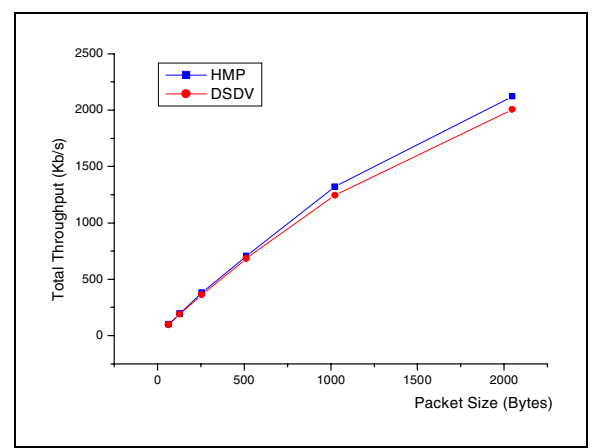

(a) Data from Routers to GW

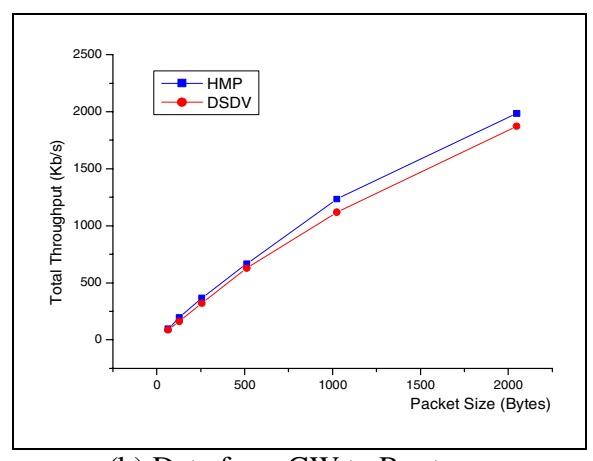

(b) Data from GW to Routers

Fig. 3. Total Throughput with Packet Size

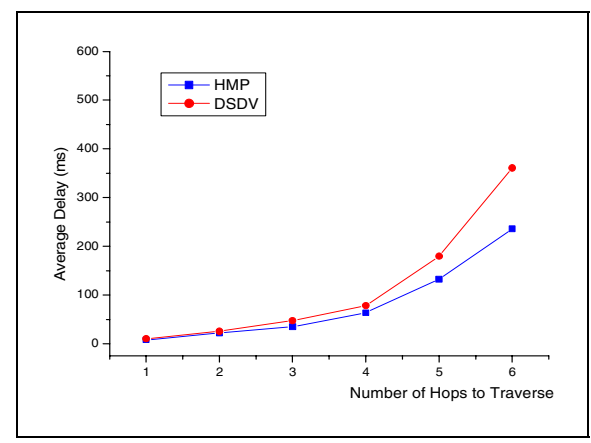

(a) Data from Routers to GW

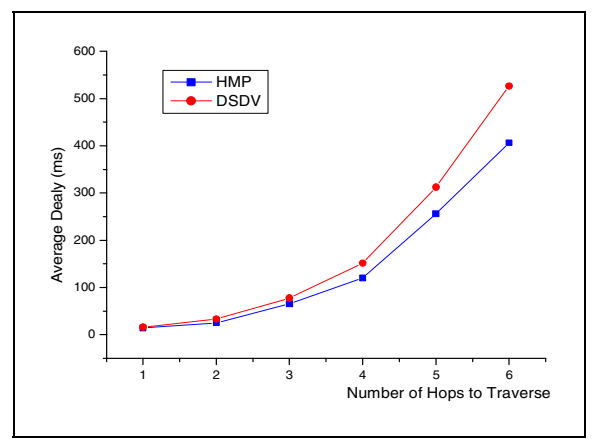

(b) Data from GW to Routers

Fig. 4. Average Delay with the Number of Hops

In figure 3, the total throughput of both HMP and DSDV increases as the size of packet is increased, but the rate of increase becomes slow. In figure 4, the average delay of both HMP and DSDV increases sharply as the hop count to traverse is increased. As the hop count to traverse is increased, the data experience more contentions in MAC layer. Therefore, we can observe that the average delay increase sharply according to the hop counts in the figure 4. Especially, the amount of data in the network is increased, the contention becomes severe. Moreover, as the amount of dada is increased in the network, each router's queue is filled with data to send or retransmit. Therefore, the total throughput is saturated showed in the figure 3 .

Figure 5 shows the total throughput according to the location and the number of mesh routers which participate in transmitting data when the size of packet is 1024 bytes. The sources or destinations are selected according to the following rules. First, remote routers from the $\mathrm{GW}$ are selected(from router 15 to router 10). Next, the mesh routers are randomly selected(from router 1 to router 15). If the randomly chosen router finishes the data transmission, the other router is randomly chosen to send data during randomly chosen transmission time to the destination. Finally, near routers to the $\mathrm{GW}$ are selected(from router 6 to router 1 ). 
We can see that HMP shows better throughput than DSDV in all cases. As the number of source or destination routers increases, the throughput gap between HMP and DSDV gets wide. We observe that the sources or destinations which are located close to the destination or source have better throughput in the figure 5 . As the distance between the source and destination is increased, the throughput is decreased. The reason why HMP shows better performance is the same as we explained early in previous figures, figure 3 and 4.

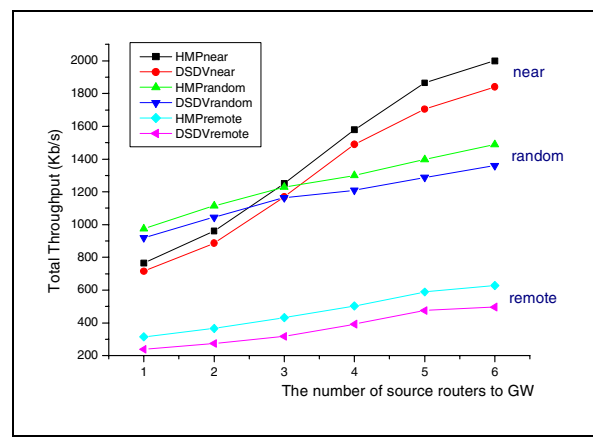

(a) Data from Routers to GW

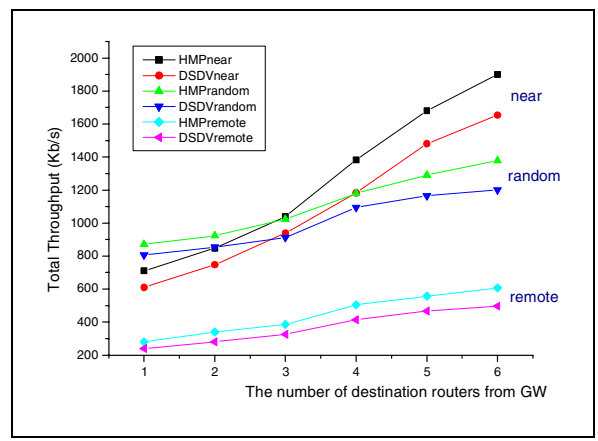

(b) Data from GW to Routers

Fig. 5. Total Throughput with Location and the Number of Routers

We can consider the hop counts from the GW as a mesh router's location. The location considerably affects network performance showed in figure 4 and 5 due to the nature of IEEE 802.11 MAC such as RTS, CTS, and ACK. The difference of throughput and delay according to the location is very large and this fact gives rise to unfairness and difficulty of the performance in order to support time-sensitive data. Therefore, the ways to guarantee QoS and fairness are necessary and very important. We will take into account these issues in the future.

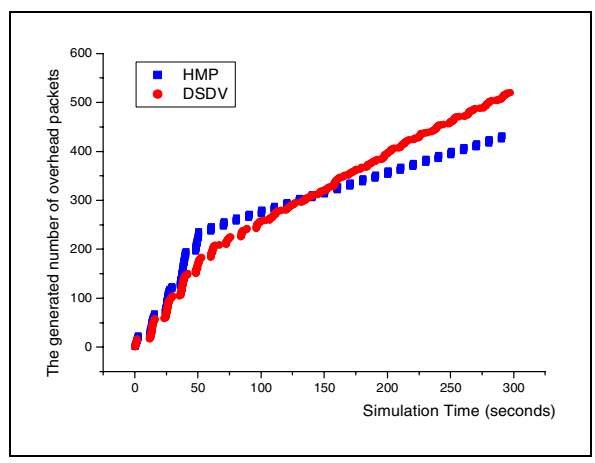

Fig. 6. Generated Overhead Packets

Figure 6 shows the generated overhead packets in the network. Because DSDV and HMP exchange routing tables before sending data, they generate lots of overhead 
packets at the beginning of simulation. However, as the simulation time increases, the overhead packets of DSDV and HMP are not increased sharply. Compared with DSDV, HMP generates more overhead packets at the beginning of simulation but it generates fewer overhead packets than DSDV with increase of the simulation time. This is because HMP generates routing table update packets and neighbor table update packets simultaneously at the beginning of simulation. However, HMP does not generate overhead packets for periodic routing table updates after getting network topology as long as the topology is not changed. After getting network topology, HMP exchanges only HELLO messages. Therefore, the rate of generating overhead packets of HMP becomes very slow.
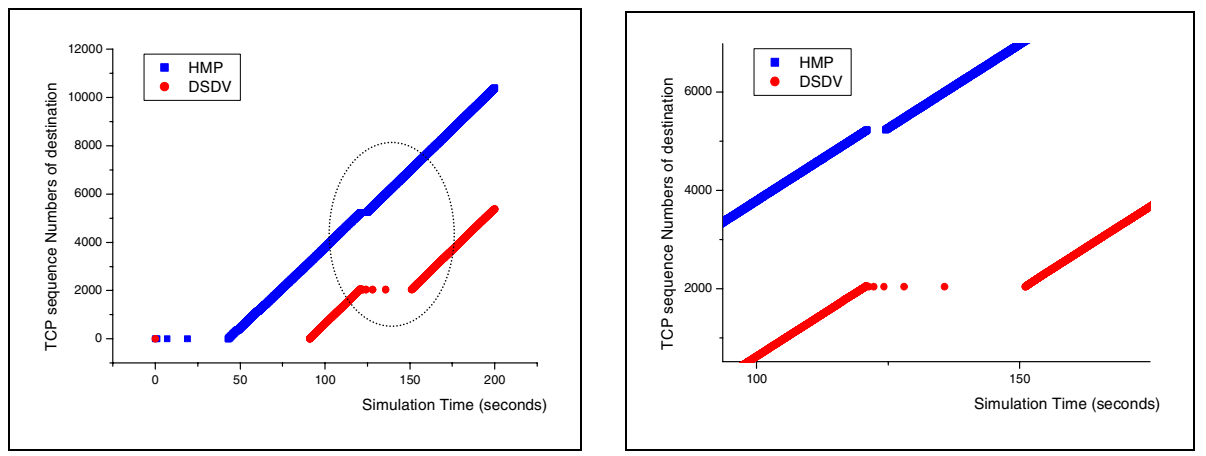

Fig. 7. Sequence Numbers of TCP

Figure 7 shows the sequence numbers of TCP in the destination. In this simulation, there are one source(router 15) and one destination $(\mathrm{GW})$. The source sends data consecutively and when it arrives 120 second, the route is failed. The right figure is the magnified version of the left one around 120 second. In the figure 7, HMP starts transmitting data faster than DSDV because HMP takes less time to know network information by modifying the routing table update scheme. Because DSDV meets several periodic routing table updates during initial forming a forwarding table, DSDV takes more time to construct a forwarding table.

After the route failure, each routing protocol detects the route failure and operates each procedure of route repairs. When a route failure is occurred, DSDV operates periodic forwarding table updates without any procedure of detection or repair. DSDV takes about 30 seconds to recover the route in the simulation, which can be identified through breaking sequence number of DSDV. When a route failure is detected through HELLO message, HMP doesn't have to update the forwarding table immediately. HMP operates the neighbor table update by setting the failed router's residual bandwidth zero and did not choose the failed router as a next hop as we proposed. HMP can be aware of the route break faster than DSDV depending on the interval of broadcasting residual bandwidth information and HMP find another path directly to the destination. In our simulation, HMP takes about 5 seconds to restart transmitting data and it detects the route failure within the maximum 10 seconds. Therefore, HMP can reduce a lot of data loss by finding and using another path to the 
destination fast and directly with multiple next hop information and the periodic HELLO message when route break is occurred.

\section{Conclusions and Future Research}

We have proposed a new hop-by-hop multipath routing scheme that is suitable for mesh routers in wireless mesh network by extending DSDV and adding the exchange of HELLO message. The proposed HMP routing protocol has two routing tables. One is a forwarding table containing multiple next hop information with minimum hop counts which makes possible to be hop-by-hop multipath. The other is a neighbor table with the neighbor routers' residual bandwidth information to determine a next hop and control the topology. Our scheme has better reliability to transmit data and it can distribute data over the network with preventing bottleneck. Through simulations by NS-2, we represented that our scheme is better than DSDV in delivering data efficiently when traffic is heavy, reducing the amount of overhead, and transmitting data when the route failure is occurred.

We represented that the performance of throughout and delay decreases sharply according to the location and the amount of traffic due to the nature of IEEE 802.11 MAC. Therefore, the ways to guarantee QoS and fairness are necessary and very important and we will extend our proposed scheme so as to provide QoS and fairness support. In this paper, we considered only mesh routers which have very low mobility in a static topology but, in the future, we will take into account the mobility of mesh routers and mesh clients in dynamic topology. Our proposed scheme is not guaranteed to be the best choice because the selected neighbor's neighbor may have very little available bandwidth. We will discuss this problem more and try to solve it. We will consider extending OLSR to a multipath scheme for wireless mesh networks.

\section{References}

1. I. F. Akyildiz, X. Wang, and W. Wang, 'Wireless Mesh Networks: A Survey" Computer Networks Journal (Elsevier), vol. 47, pp. 445-487, Mar. 2005.

2. E. M. Royer and C. K. Toh, "A Review of Current Routing Protocols for Ad Hoc Mobile Wireless Networks”, IEEE Personal Communications, vol. 6, pp. 46-55, Apr.1999.

3. X. Zou, B. Ramamurthy, and S. Magliveras "Routing Techniques in Wireless Ad Hoc Networks - Classification and Comparison", The Sixth World Multiconference on Systemics, Cybernetics, and Informatics ( SCI '02), vol. 4, Jul. 2002

4. C. E. Perkins and P. Bhagwat "Highly Dynamic Destination-Sequenced Distance-Vector Routing (DSDV) for Mobile Computers", ACM Conference on Communications Architectures, Protocols and Applications (SIGCOMM '94), pp.234-244, Aug. 1994

5. P. Jacquet, P. Muhlethaler, T. Clausen, A. Laouiti, A. Qayyum, and L. Viennot, "Optimized Link State Routing Protocol for Ad Hoc Networks", Proceedings of IEEE International Multi Topic Conference (INMIC '02), pp. 62-68, Dec. 2002

6. DSR - D. B. Johnson, D. A. Maltz, and Y. C. Hu, "The Dynamic Source Routing Protocol for Mobile Ad Hoc Networks(DSR)", Internet Draft, Apr. 2003

7. AODV - C. E. Perkins and E. M. Royer, "Ad hoc On-demand Distance Vector(AODV) Routing”, In Proceeding of Second IEEE Workshop on Mobile Computing System and Application (WMCSA '99), pp. 90-100, Feb. 1999. 
8. R. Jansen, S. Hanemann, and B. Freisleben, "Bandwidth Efficient Distance Vector Routing for Ad Hoc Networks", In Proceedings of the Wireless and Optical Communications Conference (WOC '01), pp. 117-122, Jun. 2001.

9. S. Mueller, R. P. Tsang, and D. Ghosal, "Multipath Routing in Mobile Ad Hoc Networks: Issues and Challenges", Lecture Notes in Computer Science, vol. 2965, pp. 209 - 234, Apr. 2004.

10. S. Vutukury and J.J. Garcia-Luna-Aceves, "MDVA:A Distance-vector Multipath Routing Protocol”, In Proceeding of IEEE INFOCOM (INFOCOM '01), vol. 1, pp. 557-564, Apr. 2001.

11. L. Chen and W. B. Heinzelman, "QoS-aware Routing Based on Bandwidth Estimation for Mobile Ad Hoc Networks", IEEE Journal on Selected Area in Communications (JSAC '05), vol. 23, pp. 561-572, Mar. 2005.

12. M. Gerla, R. Bagrodia, L. Zhang, K. Tang, and L. Wang, "TCP over Wireless Multi-hop Protocols: Simulation and Experiments", In Proceeding of IEE International Conference on Communications (ICC '99), Jun 1999.

13. G. Holland and N. Vaidya, "Analysis of TCP Performance over Mobile Ad Hoc Networks", In proceeding of ACM Conference of Mobile Communication (MOBICOM '99), Aug. 1999. 